play is not brilliant. The light is comparable with that of the Milky Way, and the moonlight between the first and third quarters is usually sufficient to overcome that of the aurora. Owing to this feeble light, the spectroscopic observations were not successful. On some occasions the characteristic yellow line near D was seen in a directvision spectroscope, but no record was obtained by photography, though plates were exposed from a few minutes to twenty-four hours and longer.

In the last section Commander Chetwynd and Dr. Chree discuss the results of the magnetic observations. One interesting result is the determination of the position of the south magnetic pole by the method of observed declinations and inclinations. The results are as follows:-

By declination $\quad \ldots \quad 72^{\circ} 50^{\prime} \mathrm{S}$. lat. $\quad \ldots \quad 155^{\circ} \quad 20^{\prime} \mathrm{E}$. long.

", inclination $\ldots \quad 72^{\circ} \mathbf{5 2}^{\prime} \quad, \quad \ldots \quad 156^{\circ} \quad 30^{\prime} \quad$,

The close agreement is curious and eminently satisfactory.

\section{THE NORTH OF ENGLAND EDUCATION CONFERENCE.}

THE seventh annual meeting of the above conference was held at Manchester during the latter half of last week, January $7-9$. The meeting was very well attended, and the arrangements for social intercourse and general comfort were excellent. There was a conversazione on Thursday evening at the Municipal School of Techno$\operatorname{logy}$, and a reception at the Town Hall on Friday evening. A convenient handbook was issued containing a full programme.

The actual work of the conference began on Friday, when the president, the Right Rev. Bishop Welldon, Dean of Manchester, delivered his presidential address. He put great stress on the fact that everything in education depends ultimately on the teacher, who cannot be too highly trained; but the success of education depends also on the completeness with which the good scholar can ascend the educational ladder right up to the university. The ultimate aim of all educational efforts is "to fit the young, young men and women alike, to be good husbands and wives, good parents of families, good citizens."

In his address on "The Incidence of the Cost of Education," Lord Stanley of Alderley appealed for more Government aid in the shape of money, and he suggested that a Royal Commission should be appointed in order to inquire into the cost of education. He maintained, in any case, that a large share of the cost should be borne locally by the levying of rates, whereas Alderman Oulton (Liverpool) was rather in favour of increased taxation for educational purposes. There was general agreement on one point-that more money is necessary. It is to be hoped that this suggestion made during the conference on the question of increased financial aid will bear fruit in some form or other. We want to get the best men and women into the teaching profession. They must be well trained, and should be able to expect a reasonably adequate return for the expense of their training and education. The building of modern schools and their outfitting of course also cost money, and every teacher knows how much better he can teach in a good and well equipped than in a poorly furnished and badly lighted and ventilated classroom.

In the afternoon "The Supply of Teachers" was under discussion. It appears that the supply far exceeds the demand at present, but that circumstances should be made use of to weed out inefficient teachers and replace them by better ones. "The Teaching of Languages" and "The Training of Girls in Domestic Subjects "also came in for debate during the afternoon. In the former, Prof. Sonnenschein appeared as the champion of Latin, though not to the exclusion of German and French. French might even be taken at school before Latin. In the latter, Miss Margaret Ashton argued that it would not be right that girls and boys should be educated entirely on the same lines.

On Saturday, at the general meeting. "The Coordination of the Curricula in Primary and Secondary Schools" formed the subject of a paper by Mr. Paton (Manchester Grammar School). He maintained that the teaching of science is adequately cared for in the school curricula, but that there is a lack of continuity in the teaching of classics. He mentioned the fact that at the present day most members of the Church, of the Civil Services, of the journalistic and diplomatic professions have been through mainly a classical education; and he held that therefore more attention should be paid to classical education. Most men of science, however, would arrive at the opposite conclusion. Reference may here be made to some remarks made by Lord Fitzmaurice at a recent Royal Society dinner, and quoted by Sir E. Ray Lankester in one of his essays "From an Easy Chair." "It is every day becoming more and more certain that science is the master." Lord Fitzmaurice further said that at no distant date it may be considered not only reasonable, but necessary, to replace the present-day diplomatists by men of science.

We must always remember, however, that the teaching of science at school is still a comparatively recent development. We are not only still learning and experimenting how to teach the subject as well or better than the ancient languages are taught, but we have to contend against a great deal of traditional, and perhaps not unnatural, prejudice.

In the afternoon a powerful plea was put in for the evening instruction of the so-called " masses." There is no doubt that there are really a very large number of men and women workers willing to improve their minds, not only along technical, but also along purely academic lines; but this can only to a limited extent be done by the provision of evening courses. A university degree based on an "evening-class" knowledge cannot, on the average, be so well earned as a degree depending on day work. The idea of giving full university degrees on the strength of evening work should not be encouraged; but everything should be done by universities to encourage the attendance of day workers as students at evening classes in order to cultivate their minds, without any intention of taking a degree. Scholarships obtained at evening courses might then lead on to day courses.

A detailed discussion on the subject of "Methods of Teaching Mathematics" concluded the business of the conference on Saturday afternoon. On this occasion the two chief papers were read by Mr. Garstang (Bedicles School, Petersfield) and Mr. Brotherton (School of Technology, Manchester).

\section{THE AETHER OF SPACE. ${ }^{1}$}

THIRTY years ago Clerk Maxwell gave in this plact a remarkable address on "Action at a Distance. It is reported in the Journal of the institution, vol. vii., and to it I would direct attention. Most natural philosophers hold, and have held, that action at a distance across empty space is impossible; in other words, that matter cannot act where it is not, but only where it is. The question, "Where is it?" is a further question that may demand attention and require more than a superficial answer. For it can be argued on the hydrodynamic or vortex thcory of matter as well as on the electrical theory, that every atom of matter has a universal, though nearly infinitesimal, prevalence, and extends everywhere, since there is no definite sharp boundary or limiting periphery to the region disturbed by its existence. The lines of force of an isolated electric charge extend throughout illimitable space and though a charge of opposite sign will curve and concentrate them, yet it is possible to deal with both charges, by the method of superposition, as if they each existed separately without the other. In that case, therefore, however far they reach, such nuclei clearly exert no "action at a distance" in the technical sense.

Some philosophers have reason to suppose that mind can act directly on mind without intervening mechanism, and sometimes that has been spoken of as genuine action at a distance; but, in the first place, no proper conception or physical model can be made of such a process, nor is it clear that space and distance have any particular meaning in the region of psychology. The links between mind and mind may be something quite other than 1 Abstract of discourse delivered at the Royal Institution on February 2r, r 908 , by Sir Oliver Lodge, F.R.S.

No. 2046, VOL. 79] 
physical proximity, and in denying action at a distance across empty space I am not denying telepathy or other activities of a non-physical kind; for although brain disturbance is certainly physical and is an essential concomitant of mental action, whether of the sending or receiving variety, yet we know from the case of heat that a material movement can be excited in one place at the expense of corresponding movement in another, without any similar kind of transmission or material connection between the two places; the thing that travels across vacuum is not heat.

In all cases where physical motion is involved, however, I would have a medium sought for; it may not be matter, but it must be something; there must be a connecting link of some kind, or the transference cannot occur. There can be no attraction across really empty space; and even when a material link exists, so that the connection is obvious, the explanation is not complete, for when the mechanism of attraction is understood it will be found that a body really only moves because it is pushed by something from behind. The essential force in nature is the vis a tergo. So when we have found the "traces," or discovered the connecting thread, we still run up against the word "cohesion," and ought to be exercised in our minds as to its ultimate meaning. Why the whole of a rod should follow, when one end is pulled, is a matter requiring explanation; and the only explanation that can be given involves, in some form or other, a continuous medium connecting the discrete and separated particles or atoms of matter.

When a steel spring is bent or distorted, what is it that is really strained? Not the atoms-the atoms are only displaced; it is the connecting links that are strained -the connecting medium-the xther. Distortion of a spring is really distortion of the rether. All stress exists in the xther. Matter can only be moved. Contact does not exist between the atoms of matter as we know them; it is doubtful if a piece of matter ever touches another piece, any more than a comet touches the sun when it appears to rebound from it; but the atoms are connected, as the comet and the sun are connected, by a continuous plenum without break or discontinuity of any kind. Matter acts on matter only through the æther. But whether matter is a thing utterly distinct and separate from the æther, or whether it is a specifically modified portion of it-modified in such a way as to be susceptible of locomotion, and yet continuous with all the rest of the æther, which can be said to extend everywhere-far beyond the bounds of the modified and tangible portion-are questions demanding, and I may say in process of receiving, answers.

Every such answer involves some view of the universal and possibly infinite uniform omnipresent connecting medium, the rther of space.

It has been said, somewhat sarcastically, that the æther was made in England. The statement is only an exaggeration of the truth. I might even urge that it has been largely constructed in the Royal Institution, for will remind you now of the chief lines of evidence on which its existence is believed in, and our knowledge of it is based. First of all, Newton recognised the need of a medium for explaining gravitation. In his "Optical Queries" he shows that if the pressure of this medium is less in the neighbourhood of dense bodies than at great distances from them, dense bodies will be driven towards each other, and that if the diminution of pressure is inversely as the distance from the dense body, the law will be that of gravitation.

All that is required, therefore, to explain gravity is a diminution of pressure, or increase of tension, caused by the formation of a matter unit-that is to say, of an electron or corpuscle; and although we do not yet know what an electron is-whether it be a strain centre, or what kind of singularity in the zether it may be-there is no difficulty in supposing that a slight, almost infinitesimal strain or attempted rarefaction should be produced in the ether whenever an clectron came into being to be relaxed again only on its resolution and destruction. Strictly speaking. it is not a real strain, but only a "stress," since there can be no actual yield, but only a pull or tension, extending in all directions towards infinity.
The tension required per unit of matter is almost ludicrously small, and yet in the aggregate, near such a body as a planet, it becomes enormous.

The force with which the moon is held in its orbit would be great enough to tear asunder a steel rod four hundred miles thick, with a tenacity of thirty tons per square inch, so that if the moon and earth were connected by steel instead of by gravity, a forest of pillars would be necessary to whirl the system once a month round their common centre of gravity. Such a force necessarily implies enormous tension or pressure in the medium. Maxwell calculates that the gravitational stress near the earth, which we must suppose to exist in the invisible medium, is 3000 times greater than what the strongest steel could stand, and near the sun it should be 2500 times as great as that.

The question has arisen in my mind whether, if the whole sensible universe-estimated by Lord Kelvin as equivalent to about a thousand million suns-were all concentrated in one body of specifiable density, ${ }^{1}$ the stress would not be so great as to produce a tendency towards ethereal disruption, which would result in a disintegrating explosion and a scattering of the particles once more as an enormous nebula and other fragments into the depths of space; for the tension would be a maximum in the interior of such a mass, and, if it rose to the value ${ }^{10^{33}}$ dynes per square centimetre, something would have to happen. I do not suppose that this can be the reason, but one would think there must be some reason for the scattered condition of gravitative matter.

Too little is known, however, about the mechanism of gravitation to enable us to adduce it as the strongest argument in support of the existence of an æther. The oldest valid and conclusive requisition of an ethereal medium depends on the wave theory of light, one of the founders of which was your professor of natural philn. sophy at the beginning of last century, Dr. Thomas Young.

No ordinary matter is capable of transmitting the undulations or tremors that we call light. The speed at which they go, the kind of undulation, and the facility with which they go through vacuum forbid this.

So clearly and universally has it been perceived that waves must be waves of something-something distinct from ordinary matter-that Lord Salisbury, in his presidential address to the British Association at Oxford, criticised the ather as little more than a nominative case to the verb to undulate. It is truly that, though it is also truly more than that; but to illustrate that luminiferous aspect of it, I will quote a paragraph from that lecture of Clerk Maxwell's to which I have already alluded :-

"The vast interplanetary and interstellar regions will no longer be regarded as waste places in the universe, which the Creator has not seen fit to fill with the symbols of the manifold order of His kingdom. We shall find them to be already full of this wonderful medium; so full, that no human power can remove it from the smallest portion of space, or produce the slightest flaw in its infinite continuity. It extends unbroken from star to star and when a molecule of hydrogen vibrates in the Dogstar, the medium receives the impulses of these vibrations, and after carrying them in its immense bosom for several years, delivers them, in due course, regular order, and full tale, into the spectroscope of Mr. Huggins, at Tulse Hill." (It is pleasant to remember that those veteran investigators Sir William and Lady Huggins are still at work.)

This will suffice to emphasise the fact that the eye is truly an ethereal sense-organ-the only one which we possess, the only mode by which the æther is enabled to appeal to us, and that the detection of tremors in this medium-the perception of the direction in which they go, and some inference as to the quality of the object which has emitted them-cover all that we mean by "sight " and "secing."

I pass, then, to another function, the electric and magnetic phenomena displayed by the rther, and on this I will only permit myself a very short quotation from the

1 On doing the Arithmetic, however, I find the necescary concentration absurdly great, showing that such a mass is quite insufficient.

No. 2046, VOL. 79] 
writings of Faraday, whose whole life may be said to have been directed towards a better understanding of these cthereous phenomena. Indeed, the statue in your entrance hall may be considered as the statue of the discoverer of the electric and magnetic properties of the æther of space.

Faraday conjectured that the same medium which is concerned in the propagation of light might also be the agent in electromagnetic phenomena. "For my own part," he says, "considering the relation of a vacuum to the magnetic force, and the general character of magnetic phenomena external to the magnet, I am much more inclined to the notion that in the transmission of the force there is such an action, external to the magnet, than that the effects are merely attraction and repulsion at a distance. Such an action may be a function of the æther for it is not unlikely that, if there be an æther, it should have other uses than simply the conveyance of radiation."

This conjecture has been amply strengthened by subsequent investigations.

One more function is now being discovered; the æther is being found to constitute matter-an immensely interesting topic, on which there are many active workers at the present time. I will make a brief quotation from your present professor of natural philosophy (J. J. Thomson), where he summarises the conclusion which we all see looming before us, though it has not yet been completely attained, and would not by all be similarly expressed :-

"The whole mass of any body is just the mass of æther surrounding the body which is carried along by the Faraday tubes associated with the atoms of the body. In fact, all mass is mass of the æther; all momentum, momentum of the æther; and all kinetic energy, kinetic energy of the æther. This view, it should be said, requires the density of the æther to be immensely greater than that of any known substance."

Yes, far denser-so dense that matter by comparison is like gossamer, or a filmy, imperceptible mist, or a Milky Way. Not unreal or unimportant-a cobweb is not unreal, nor to certain creatures is it unimportant, but it cannot be said to be massive or dense; and matter, even platinum, is not dense when compared with the æther. Not until last year, however, did I realise what the density of the æether must really be, ${ }^{1}$ compared with that modification of it which appeals to our senses as matter, and which for that reason engrosses our attention. If I have time I will return to that before I have finished.

Is there any other function possessed by the rther which, though not yet discovered, may lie within the bounds of possibility for future discovery? I believe there is, but it is too speculative to refer to, beyond saying that it has been urged as probable by the authors of "The Unseen Universe," and has been thus tentatively referred to by Clerk Maxwell :-

"Whether this vast homogeneous expanse of isotropic matter is fitted not only to be a medium of physical interaction between distant bodies, and to fulfil other physical functions of which, perhaps, we have as yet no conception, but also .... to constitute the material organism of beings exercising functions of life and mind as high or higher than ours are at present-is a question far transcending the limits of physical speculation."

And there, for the present, I leave that aspect of the subject.

I shall now attempt to illustrate some relations between æther and matter.

The question is often asked, Is æther material? This is largely a question of words and convenience. Undoubtedly the æther belongs to the material or physical universe, but it is not ordinary matter. I should prefer to say it is not "matter" at all. It may be the substance or substratum or material of which matter is composed, but it would be confusing and inconvenient not to be able to discriminate between matter, on the one hand, and æther on the other. If you tie a knot on a bit of string, the knot is composed of string, but the string is not composed of knots. If you have a smoke or vortexring in the air, the vortex-ring is made of air, but the atmosphere is not a vortex-ring, and it would be only confusing to say that it was.

$$
\text { See Lodge, Phil. Mag., April, rşo7. }
$$

No. 2046 , VOL. 79]
The essential distinction between matter and ather is that matter moves, in the sense that it has the property of locomotion and can effect impact and bombardment, while æther is strained, and has the property of exerting stress and recoil. All potential energy exists in the æether. It may vibrate, and it may rotate, but as regards locomotion it is stationary-the most stationary body we know-absolutely stationary, so to speak; our standard of rest.

All that we ourselves can effect, in the material universe, is to alter the motion and configuration of masses of matter; we can move matter by our muscles, and that is all we can do directly; everything else is indirect.

But now comes the question, How is it possible for matter to be composed of ather? How is it possible for a solid to be made out of fluid? A solid possesses the properties of rigidity, impenetrability, elasticity, and such like; how can these be imitated by a perfect fluid such as the rther must be? The answer is, they can be imitated by a fluid in motion, a statement which we make with confidence as the result of a great part of Lord Kelvin's work.

It may be illustrated by a few experiments.

A wheel of spokes, transparent or permeable when stationary, becomes opaque when revolving, so that a ball thrown against it does not go through, but rebounds. The motion only affects permeability to matter; transparency to light is unaffected, until something near the speed of light itself is reached.

A silk cord hanging from a pulley becomes rigid and viscous when put into rapid motion, and pulses or waves which may be generated on the cord travel along it with a speed equal to its own velocity, whatever that velocity may be, so that they appear to stand still. This is a case of kinetic rigidity, and the fact that the wave-transmission velocity is equal to the rotatory speed of the material is typical and important, for in all cases of kinetic elasticity these two velocities are of the same order of magnitude.

A flexible chain, set spinning, can stand up on end while the motion continues.

A jet of water at sufficient speed can be struck with a hammer, and resists being cut with a sword.

A spinning disc of paper becomes elastic, like flexible metal, and can act like a circular saw. Sir William White tells me that in naval construction steel plates are cut by a rapidly revolving disc of soft iron.

A vortex-ring, ejected from an elliptical orifice, oscillates about the stable circular form, as an india-rubber ring would do, thus furnishing a beautiful example of kinetic elasticity, and showing us clearly a fluid displaying some of the properties of a solid.

A still further example is Lord Kelvin's model of a spring balance, made of nothing but rigid bodies in spinning motion. ${ }^{1}$

If the æther can be set spinning, therefore, we may have some hope of making it imitate the properties of matter, or even of constructing matter by its aid. But how are we to soin the æther? Matter alone seems to have no grip of it. I have spun steel discs, a yard in diameter, 4000 times a minute, have sent light round and round between them, and tested carefully for the slightest effect on the rther. Not the slightest effect was perceptible. We cannot spin æther mechanically.

But we can vibrate it electrically, and every source of radiation does that. An electrified body, in sufficiently rapid vibration, is the only source of ather-waves that we know, and if an electric charge is suddenly stopped it generates the pulses known as X-rays, as the result of the collision. Not speed, but sudden change of speed, is the necessary condition for generating waves in the xther by electricity.

We can also infer some kind of rotary motion in the æther, though we have no such obvious means of detecting the spin as is furnished by vision for detecting some kinds of vibration. It is supposed to exist whenever we put a charge into the neighbourhood of a magnetic pole. Round the line joining the two the xther is spinning like a top. I do not say it is spinning fast: that is a question of its density: it is, in fact, spinning with excessive slowness, but it is spinning with a definite moment of momentum.

1 Address to Section A of British Association at Montreal, 1884. 
J. J. Thomson's theory makes its moment of momentum exactly equal to em, the product of charge and pole, the charge being measured electrostatically and the pole magnetically.

How can this be shown experimentally? Suppose we had a spinning top enclosed in a case, so that the spin was unrecognisable by ordinary means-it could be detected by its gyrostatic behaviour to force. If allowed to "precess" it will respond by moving perpendicularly to a deflecting force. So it is with the charge and the magnetic pole. Try to move the charge suddenly, and it immediately sets off at right angles. A moving charge is a current, and the pole and the current try to revolve round one another-a true gyrostatic action due to the otherwise unrecognisable ethereal spin. The fact of such magnetic rotation was discovered by Faraday.

I know that it is usually worked out in another way, in terms of lines of force and the rest of the circuit; but I am thinking of a current as a stream of projected charges, and no one way of regarding such a matter is likely to exhaust the truth or to exclude other modes which are equally valid. Anyhow, in whatever way it is regarded, it is an example of the three rectangular vectors.

The three vectors at right angles to each other, which may be labelled current, magnetism, and motion respectively, or more generally $\mathrm{E}, \mathrm{H}$, and $\mathrm{V}$, represent the quite fundamental relation between wher and matter, and constitute the link between electricity, magnetism, and mechanics. Where any two of these are present, the third is a necessary consequence. This principle is the basis of all dynamos, of electric motors, of light, of telegraphy, and of most other things. Indeed, it is a question whether it does not underlie everything that we know in the whole of the physical sciences, and whether it is not the basis of our conception of the three dimensions of space.

Lastly, we have the fundamental property of matter called inertia, which, if I had time, I would show could be explained electromagnetically, provided the ethereal density is granted as of the order $10^{12}$ grams per cubic centimetre. The elasticity of the æether would then have to be of the order $10^{33}$ C.G.S., and if this is due to intrinsic turbulence, the speed of the whirling or rotational elasticity must be of the same order as the velocity of light. This follows hydrodynamically, in the same sort of way as the speed at which a pulse travels on a flexible running endless cord, the tension of which is entirely due to the centrifugal force of the motion, is precisely equal to the velocity of the cord itself; and so, on our present view, the intrinsic energy of constitution of the æther is incredibly and portentously great, every cubic millimetre of space possessing what, if it were matter, would be a mass of a thousand tons, and an energy equivalent to the output of a million-horse-power-station for forty million years.

The universe we are living in is an extraordinary one, and our investigation of it has only just begun. We know that matter has a psychical significance, since it can constitute brain, which links together the physical and the psychical worlds. If anyone thinks that the æther, with all its massiveness and energy, has probably no psychical significance, I find myself unable to agree with him.

\section{SCIENCE AND THE PRACTICAL PROBLEMS} OF THE FUTURE. ${ }^{1}$

$\mathrm{A}^{\mathrm{T}}$ the recent conference on the conservation of resources which met at the White House at the invitation of the President of the United States, notes of warning were sounded concerning the coming exhaustion of coal, wood, ores, and soils. Whether or not we accept as exact the 'stimates furnished by experts on that impressive occasion, there is no doubt that we are approaching the end of our available resources, and that the near future will have momentous problems to face.

Certain things are clear.

First.-Unchecked wastefulness as exhibited, for example, in the extermination of the bison, in the destruction of forests, in the exhaustion of the soil, in the disappear-

1 Abridged from the address delivered by Prof. F. L. Nichols, the retiring the Baltimore meeting, December, rgo8.

No. 2046, VOL. 79] ance from our coasts and streams, that once teemed with fish, of this important source of food supply, in the pouring into the air of an incredible amount of unused fuel from hundreds of thousands of coke ovens, must cease, or our ruthless exploitation will bring disaster on generations soon to come. The prevention of these and countless other manifestations of individual and corporate greed is a problem for the economist and the law-maker, although they will scarcely succeed in its solution without calling science to their aid.

Second.-Saving and thrift offer at best only a postponement of the day of distress. The end of our supplies of coal and petroleum must ultimately be reached. Forests may be renewed and the soil restored to its maximum fertility, but the problem which is presently to confront the race is that of civilised existence without recourse to energy stored by the slow processes of nature. This problem must be definitely solved before the complete exhaustion of our inherited capital.

Third.-The problem is not without conceivable solution, since the annual accession of energy from the sun, did we know how to utilise it without awaiting the slow processes of storage employed by nature, is ample for every thinkable need of the future inhabitants of our planet. Estimates of the constant of solar radiation show that about $2 \cdot 18$ kilowatts of power is continually received from the sun for every square metre of the earth's surface, or more than seven and a half millions of horse-power per square mile. The present use of power in the United States is about eighty million horse-power, or one horsepower per capita. This quantity is likely to increase more rapidly than the population in the future unless curtailed by lack of fuel, but it is evident that a very small fraction of the sun's radiation would meet all demands.

Now abundant power is soon to be the factor upon which material advancement will chiefly depend. To obtain it in the face of the disappearance of coal and petroleum will be imperative. For success in this, upon which in the immediate future the welfare of the race and ultimately its very perpetuity is to depend, we must look to science. Mere ingenuity or inventiveness, however widely developed, will not suffice. The inventor and the engineer can but utilise and apply the material which the man of science provides, and with the exhaustion of our stores of scientific knowledge civilisation must halt.

It is of this fundamental relation of science to the progress of our civilisation that I wish to speak. The fact that material progress is based upon science seems to be but dimly understood. It appears to be generally supposed that it is to the inventor and to those who use his devices that we owe our present advantages over our forefathers. I would not belittle the achievements of the socalled practical man, but the public must be taught that application can never run ahead of the knowledge to be applied, and that the only road to higher achievement in practical things is by the further development of pure science.

The main product of science, using that word in its broadest sense, is knowledge; among its by-products are the technological arts, including invention, engineering in all its branches, and modern industry. Not all industries have attained the character of a technological art. Burning the woods to drive out game, and thus obtain a dinner, is a form of industry. Like it in character are some very large industries, such as agriculture of the sort that impoverishes the soil; lumbering that destroys forests, and incidentally ruins rivers and increases erosion; cokemaking by processes that waste 40 per cent. of the energy of coal. The production of power from coal by means of the steam boiler and the reciprocating engine we at present regard as a highly developed technological art; yet it is a process which, at the very best, converts less than ro per cent. of the total stored energy of the fuel into available form. If the ultimate purpose of this power is the production of light, we by our present methods suffer a second waste of 90 per cent. or more, so that the efficiency of the combined processes is but a fraction of I per cent. These things are excusable while ignorance lasts. They become criminal with realisation of the results, and are inconceivable in a community of fully developed civilisation. Science paves the way for the gradual sup- 